Vol. 38 (1988) [335-338]

\title{
CHARACTERISATION OF FUNCTIONAL PROPERTIES BY NETS
}

\author{
Carlos R. Borges
}

\begin{abstract}
We characterise continuous closed (perfect, open) functions in terms of nets. This enables us to improve some significant results, with much simpler proofs.
\end{abstract}

The primary motivation for this study is the following result of Bourbaki, a nice proof of which appears in Lemma 14 of [2]. Let $X, Y$ and $Z$ be Tychonoff spaces. If $f: X \rightarrow Y$ and $g: Y \rightarrow Z$ are continuous onto maps such that $h=g f$ is perfect, then $f$ and $g$ are perfect.

The preceding result has played a major rôle in the proof of several significant results, but its proof is surely nontrivial and requires the machinery of compactifications. Our characterisation of closed continuous functions allows us to prove this result in a more general setting, with little effort. There are other gains.

TheOREM 1. Let $f: X \rightarrow Y$ be a continuous map from $X$ onto $Y$. Then $f$ is a closed map if and only if, for each $y \in Y$, net $\left\{y_{\nu}\right\}_{\nu \in \wedge}$ in $Y$ which converges to $y$ and any choice of $x_{\nu} \in f^{-1}\left(y_{\nu}\right),\left(\left\{x_{\nu} \mid \nu \in \wedge\right\}\right)^{-} \cap f^{-1}(y) \neq \emptyset$.

Próof: The "only if" part. Suppose $f$ is closed and $\lim _{\nu} y_{\nu}=y, \nu \in \wedge$. Pick $x_{\nu} \in$ $f^{-1}\left(y_{\nu}\right)$, for each $\nu \in \wedge$, and let $A=\left\{x_{\nu} \mid \nu \in \wedge\right\}$. Assuming that $\bar{A} \cap f^{-1}(y)=\emptyset$, we then get that $y \notin f(\bar{A})=\overline{f(A)}$ (because $f$ is closed), which contradicts $\lim _{\nu} y_{\nu}=y$.

The "if" part. Assume $A$ is a closed subset of $X$ but $f(A)$ is not a closed subset of $Y$. Pick $y \in \overline{f(A)}-f(A)$ and net $\left\{y_{\nu}\right\}_{\nu \in \Lambda}$ in $f(A)$ such that $\lim _{\nu} y_{\nu}=y$. For each $\nu \in \wedge$, pick $x_{\nu} \in f^{-1}\left(y_{\nu}\right) \cap A$. Then $\left(\left\{x_{\nu} \mid \nu \in \wedge\right\}\right)^{-} \subseteq A$ which implies that $\left(\left\{x_{\nu} \mid \nu \in \wedge\right\}\right) \cap f^{-1}(y)=\emptyset$, which contradicts the hypothesis.

Theorem 2. Let $f$ be a continuous map from $X$ onto $Y$. Then $f$ is a perfect map if and only if, for each $y \in Y$, net $\left\{y_{\sigma}\right\}_{\sigma \in \Gamma}$ in $Y$ which converges to $y$ and any choice of $x_{\sigma} \in f^{-1}\left(y_{\sigma}\right),\left\{x_{\sigma}\right\}_{\sigma \in \Gamma}$ has a cluster point in $f^{-1}(y)$.

Proof: The "only if" part. Let us assume that $\left\{x_{\sigma}\right\}_{\sigma \in \Gamma}$ does not have a cluster point in $f^{-1}(y)$. Then, for each $x \in f^{-1}(y)$, there exists an open neighbourhood $U_{x}$ of $x$ in $X$ and some $\beta_{x} \in \Gamma$ such that $x_{\nu} \notin U_{x}$ for all $\nu>\beta_{x}, \nu \in \Gamma$. Since $f^{-1}(y)$ is

Received 13 January, 1987

Copyright Clearance Centre, Inc. Serial-fee code: $0004-9729 / 88 \$ A 2.00+0.00$. 
compact, let $U_{x_{1}}, \ldots, U_{x_{n}}$ cover $f^{-1}(y)$. Pick $\gamma \in \Gamma$ such that $\gamma>\beta_{x_{1}}, \ldots, \gamma>\beta_{x_{n}}$. Since $f$ is closed there exists an open neighbourhood $U$ of $y$ such that $f^{-1}(U) \subseteq$ $U_{x_{1}} \cup \ldots \cup U_{x_{n}}$. It follows that $A=\left(\left\{x_{\nu} \mid \nu \in \Gamma, \nu>\gamma\right\}\right)^{-} \subseteq X-f^{-1}(U)$, which implies that $y \notin\left(\left\{y_{\nu} \mid \nu \in \Gamma, \nu>\gamma\right\}\right)^{-}=f(A)$, a contradiction (because $\lim _{\sigma} y_{\sigma}=y$ ).

The "if" part. We get immediately from Theorem 1 that $f$ is a closed map. Therefore, we need only show that each $f^{-1}(y)$ is compact: suppose not. Pick $p \in Y$ such that $f^{-1}(p)$ is not compact. Let $\left\{x_{\nu}\right\}_{\nu \in \wedge}$ be a net in $f^{-1}(p)$ which has no cluster point in $f^{-1}(p)$. Then, letting $y_{\nu}=p$, for all $\nu \in \wedge$, we get a net $\left\{y_{\nu}\right\}_{\nu \in \wedge}$ in $Y$ which converges to $p$ and points $x_{\nu} \in f^{-1}\left(y_{\nu}\right)$ such that $\left\{x_{\nu}\right\}_{\nu \in \wedge}$ has no cluster point in $f^{-1}(p)$, a contradiction. This completes the proof.

TheOREM 3. Let $f$ be a continuous map from $X$ onto $Y$. Then $f$ is open if and only if, for each $y \in Y, x \in f^{-1}(y)$ and net $\left\{y_{\nu}\right\}_{\nu \in \wedge}$ in $Y$ which converges to $y$, there exists a net contained in $f^{-1}\left(\left\{y_{\nu} \mid \nu \in \wedge\right\}\right)$ which converges to $x$.

Proof: The "only if" part. Let $\left\{N_{\alpha}(x) \mid \alpha \in D\right\}$ be an open neighbourhood base for $x \in X$ and direct $D$ by inclusion (that is, $\alpha<\beta \leftrightarrow N_{\alpha}(x) \supseteq N_{\beta}(x)$ ). Since $f$ is open and continuous, we get that $\left\{f\left(N_{\alpha}(X)\right) \mid \alpha \in D\right\}$ is an open neighbourhood base for $y \in Y$. For each $\alpha \in D$, pick $\nu_{\alpha} \in \wedge$ such that $y_{\nu_{\alpha}} \in f\left(N_{\alpha}(X)\right)$. (It can happen that there are $\alpha, \beta \in \wedge$ such that $\alpha \neq \beta$ but $\nu_{\alpha}=\nu_{\beta}$; see Proposition 4.) Next, for each $\alpha \in D$, pick $x_{\alpha} \in N_{\alpha}(X) \cap f^{-1}\left(y_{\nu_{\alpha}}\right)$ and note that $\lim _{\alpha} x_{\alpha}=x$. Clearly the net $\left\{x_{\alpha}\right\}_{\alpha \in D}$ satisfies all requirements.

The "if" part. Suppose $U \neq \emptyset$ is an open subset of $X$ such that $f(U)$ is not open in $Y$. Pick $y \in f(U)$ such that $y \in(Y-f(U))^{-}$; then pick a net $\left\{y_{\nu}\right\}_{\nu \in \wedge}$ in $Y-f(U)$ such that $\lim _{\nu} y_{\nu}=y$. Next, pick $x \in f^{-1}(y) \cap U$ and, by hypothesis, pick a net $\left\{x_{\alpha}\right\}_{\alpha \in D}$ contained in $f^{-1}\left(\left\{y_{\nu} \mid \nu \in \wedge\right\}\right)$ such that $\lim _{\alpha} x_{\alpha}=x$; note that this contradicts " $x \in U$ and $U \cap f^{-1}\left(\left\{y_{\nu} \mid \nu \in \wedge\right\}\right)=\emptyset$." This completes the proof.

Proposition 4. Let $f$ be a continuous map from $X$ onto $Y$. The following conditions are sufficient but not necessary for $f$ to be an open map: for each $y \in Y$, $x \in f^{-1}(y)$ and net $\left\{y_{\nu}\right\}_{\nu \in \wedge}$ which converges to $y$, there exists $x_{\nu} \in f^{-1}\left(y_{\nu}\right)$ such that $x \in\left(\left\{x_{\nu} \mid \nu \in \wedge\right\}\right)^{-}$or $x$ is a cluster point of $\left(\left\{x_{\nu}\right\}_{\nu \in \wedge}\right)$.

Proof: The conditions are sufficient for $f$ to be open; suppose not. Let $U \neq \emptyset$ be an open subset of $X$ such that $f(U)$ is not open in $Y$. Pick $y \in f(U)$ such that $y \in(Y-f(U))^{-}$; then pick a net $\left\{y_{\nu}\right\}_{\nu \in \wedge}$ in $Y-f(U)$ such that $\lim _{\nu} y_{\nu}=y$. Next, pick $x \in f^{-1}(y) \cap U$ and, by hypothesis, pick $x_{\nu} \in f^{-1}\left(y_{\nu}\right)$, for each $\nu \in \wedge$ such that $x \in\left(\left\{x_{\nu} \mid \nu \in \wedge\right\}\right)^{-}$( or $x$ is a cluster point of $\left.\left\{x_{\nu}\right\}_{\nu \in \wedge}\right)$; note that this contradicts " $x \in U$ and $U \cap f^{-1}\left(\left\{y_{\nu} \mid \nu \in \wedge\right\}\right)=\emptyset . "$ This shows that $f$ is open.

The conditions are not necessary for $f$ to be open: Let $N$ denote the positive 
integers and $X=\{(0,0)\} \cup\{(m, n) \mid m, n \in N\}$. Let $\tau_{1}$ be the topology for $X$ generated by the following collection $B_{1}$ of subsets of $X:$ if $(m, n) \in X-\{(0,0)\}$ then $\{(m, n)\} \in \mathcal{B}_{1}$. If $(0,0) \in U$ then $U \in B_{1}$ provided that $U$ contains all but a finite number of elements of all but a finite number of columns of $X$ (that is, for $(m, n) \in$ $X-\{0,0\},\{(m, j) \mid j \in N\}$ is the column through $(m, n))$. Let $Y=\{0\} \cup\{m \mid m \in N\}$ and let $\tau_{2}$ be the topology for $Y$ generated by the following collection $\mathcal{B}_{2}$ of subsets of $Y:$ if $m \neq 0$ and $m \in X$, then $\{m\} \in \mathcal{B}_{2}$. If $0 \in U$ then $U \in \mathcal{B}_{2}$ provided that $Y-U$ is a finite set.

Now, let $f: X \rightarrow Y$ be the map defined by $f((m, n))=m$. It is easily seen that $f$ is continuous and open. However, letting $y=0, x=(0,0) \in f^{-1}(y)$ and choosing the sequence $\{m\}_{m \in \omega}$ which converges to $y$ in $\left(Y, \tau_{2}\right)$, we easily see that if we choose any $x_{m} \in f^{-1}(m)$, for $m=1,2, \ldots$, then $x \notin\left(\left\{x_{m} \mid m=1,2, \ldots\right\}\right)^{-}$(hence $x$ is not a cluster point of $\left.\left\{x_{m}\right\}_{m \in \omega}\right)$. This completes the proof.

Theorem 5. Let $X, Y$ and $Z$ be topological spaces such that $Y$ is Hausdorff. If $f: X \rightarrow Y$ and $g: Y \rightarrow Z$ are continuous onto functions such that $h=g f$ is perfect, then $f$ and $g$ are perfect.

ProOF: The argument consists of several parts.

(i) $g$ is closed; note that, for each $A \subseteq Y, h f^{-1}(A)=g(A)\left(h f^{-1}(A)=\right.$ $\left.(g f) f^{-1}(A)=g\left(f f^{-1}\right)(A)=g(A)\right)$. Consequently, if $A$ is a closed subset of $Y$, we immediately get that $g(A)=h f^{-1}(A)$ is closed.

(ii) $f^{-1}(y)$ is compact, for each $y \in Y$ : note that $h^{-1} g(y)$ is compact and $f^{-1}(y)$ is a closed subspace of $h^{-1} g(y)$.

(iii) $g^{-1}(z)$ is compact, for each $z \in Z$ : note that $h^{-1}(z)=f^{-1} g^{-1}(z)$ is compact and $g^{-1}(z)=f h^{-1}(z)$.

(iv) $f$ is closed: (we use Lemma 2). Let $y \in Y$ and a net, $\left\{y_{\nu}\right\}_{\nu \in \wedge}$ in $Y$ which converges to $y$. Pick any $x_{\nu} \in f^{-1}\left(y_{\nu}\right)$, for each $\nu \in \wedge$. By Lemma 2, $\left\{x_{\nu}\right\}_{\nu \in \wedge}$ converges to some point $x$ in $h^{-1} g(y)$ (note that $\left\{g\left(y_{\nu}\right)\right\}_{\nu \in \wedge}$ is a net in $Z$ which converges to $g(y)$ in $Z$ and, for all $\left.w \in Y, f^{-1}(w) \subseteq h^{-1} g(w)\right)$. From the continuity of $f$, it follows that $x \in f^{-1}(y)$. (Suppose not. Then $y \neq f(x)$ with $\lim _{\nu} f\left(x_{\nu}\right)=y$ and also $\lim _{\nu} f\left(x_{\nu}\right)=f(x)$, a contradiction, since $Y$ is Hausdorff.)

From (i) through (iv) we get immediately that $f$ and $g$ are perfect, which completes the proof.

The following result also improves a very useful result (see Theorem 2.5 on p. 227 of $[\mathbf{1}])$, with a simpler proof.

TheOREM 6. Let $X$ be compact, $Y$ any space and $p: X \times Y \rightarrow Y$ be defined by 
$p(x, y)=y$. Then $p$ is perfect.

Proof: Clearly, each $p^{-1}(y)=X \times\{y\}$ is compact. Also, for any net $\left\{y_{\nu}\right\}_{\nu \in \wedge}$ in $Y$ converging to $y \in Y$ and any $z_{\nu} \in X, \nu \in \wedge$, we get immediately $\left\{\left(x_{\nu}, y_{\nu}\right)\right\}_{\nu \in \wedge}$ has a cluster point $(x, y)$ where $x$ is a cluster point for $\left\{x_{\nu}\right\}_{\nu \in \wedge}$ in $X$. By Theorem $1, p$ is closed, which completes the proof.

\section{REFERENCES}

[1] J. Dugundji, Topology (Allyn and Bacon, Boston, 1966).

[2] V.I. Ponomarev, 'On spaces co-absolute with metric spaces', Russian Math. Surveys 21 (1966), 87-114.

Department of Mathematics,

University of California,

Davis, CA 95616

United States of America. 